\title{
On Demand Uncertainty in the Newsvendor Model
}

\author{
R. Andrew Butters*
}

September 28, 2019

\begin{abstract}
I provide three comparative statics involving the level of demand uncertainty for the newsvendor model, two of which lead to robust predictions. I show that for distributions of demand that are greater in the dispersive order, both the expected (censored) sales and share of inventory sold fall. These monotone comparative statics occur despite the lack of one for the (optimal) inventory choice.
\end{abstract}

Keywords: demand uncertainty, newsvendor model, dispersive order, inventory, monotone comparative statics, censored demand

JEL: D81, D21, C60

*Business Economics and Public Policy, Kelley School of Business, Indiana University, 1309 E. Tenth Street, Bloomington, IN, 47405, USA, rabutter@indiana.edu 


\section{Introduction}

For inventory decisions under uncertainty, the newsvendor model represents the work-horse framework in both economics and operations management. The classic set-up involves making a decision under uncertainty about how much of a resource to have on hand-leading to potential costs of having "too few" or "too many," once the exact amount needed is known. Originally discussed by Edgeworth (1888) in the context of how much cash reserves a bank should have on hand, and further developed by Arrow et al. (1951), the newsvendor model has implications for settings including supply chain contracts, capacity management, and staff sizing for service industries. Consequently, the newsvendor model and its insights have been utilized in industries from apparel retail (e.g., Fisher and Raman (1996)) to the health care industry (e.g., Olivares et al. (2008)).

An important step in applying the newsvendor model to empirical settings is the derivation of comparative static predictions of endogenous variables to changes in exogenous conditions. A natural set of predictions from the newvendor model involve how changes in the level of demand uncertainty lead to changes in inventories and units sold. The usefulness of these predictions for empirical work is predicated on them requiring a minimal amount of parametric assumptions-which can be at odds with the empirical patterns present in most data. For this reason, robust monotone comparative statics that can be generated without making restrictive concavity and/or differentiability assumptions are the most valuable for empirical work (Athey, 2002).

Towards these ends, I use the dispersive order condition (Saunders, 1978; Saunders and Moran, 1978; Shaked, 1982) to rank the level of demand uncertainty, and provide three comparative statics. I show that for distributions of demand that have the same mean but are greater in the dispersive order, both the expected sales and share of inventory sold fall. These robust monotone comparative statics occur despite the fact that the (optimal) inventory choice can increase or decrease as a consequence of more uncertain demand.

These results contribute to a couple of strands of the literature on the newsvendor model. First, the results contribute to the efforts of estimating the underlying parameters associated with the newsvendor model (e.g., Gaynor and Anderson (1995), Fisher and Raman (1996), Olivares et al. (2008)) by providing robust predictions that can serve as additional moments in estimation. The results also contribute to the literatures exploring how either (i) partial information of the demand distribution (Andersson et al. 
(2013), O’Neil et al. (2016), Natarajan et al. (2017), Ninh et al. (2019)), or (ii) alternative risk preferences/behavioral biases of the newsvendor (Eeckhoudt et al. (1995), Herweg (2013), Kirshner and Shao (2019)) affect the traditional analysis. The results presented here indicate that ranking uncertainty using the dispersive order is likely to complement the efforts of generating robust predictions in other information/behavioral settings.

\section{The newsvendor model}

The newsvendor's problem involves choosing an inventory level in the face of stochastic demand. ${ }^{1}$ At the beginning of the period, the newsvendor decides on the amount of units to buy, $q$, at a per unit wholesale price of $w^{2}{ }^{2}$ The newsvendor makes this decision before observing the level of demand at the fixed retail price, $p .^{3}$ The newsvendor balances the trade-off of buying units that might go unsold (and become worthless), with the lost opportunity of selling units at the retail price. The newsvendor model can also incorporate the possibility of the newsvendor being able to sell back any unsold units at some scrap value less than the wholesale price. Adding this feature would not change any of the results of the paper, only the form of the critical fractile to be defined shortly. Demand, $x$, is assumed to be a non-negative random variable that is distributed according to the continuously differentiable cumulative distribution function, $F(x)$, defined over the nonnegative portion of the real line. While demand is stochastic, I assume the newsvendor knows

\footnotetext{
${ }^{1}$ The newsvendor model presented here has similarities to the uncertain-sequentialtrade (UST) models (e.g., Eden (1990), and Dana (1999)) which are extensions of the model of Prescott (1975).

${ }^{2}$ Under the stated conditions regarding costs (linear), the model can be extended to include a level of "current" inventory, without changing the main comparative statics of the paper.

${ }^{3}$ Settings in which the newsvendor also endogenously chooses the price(s) before the realization of demand have been explored ever since Whitin (1955), (e.g., Petruzzi and Dada (1999)). Salinger and Ampudia (2011) show the Lerner relationship linking the optimal price to the marginal cost and price elasticity of demand can be generalized to the (singe) price-setting newsvendor. Dana (1999) characterizes a set of conditions in the environment where the firm(s) selects a distribution of prices. The assumption of an exogenous fixed price is consistent with the empirical evidence provided by (DellaVigna and Gentzkow, 2019), and facilitates the tight connection between the measurement of uncertainty and its impact on endogenous variables.
} 
the distribution $F(x){ }^{4}$

The total number of units sold is censored by the inventory decision. For realizations of $x>q$, the number of units sold is $q$, while for realizations $x \leq q$, the number of units sold is $x$, leaving $q-x$ units unsold. Conditional on a inventory choice, $q$, and the distribution of demand, $F$, denote the expected number of units sold to be: $\bar{x}_{q, F}=\int_{0}^{\infty} \min \{x, q\} \partial F(x)$, where at times the $q$ subscript is suppressed.

Assumption 1. The retail price and wholesale price are such that $0<w<$ $p$.

Assumption 1 ensures that the newsvendor can sell each unit demanded at a positive margin, and ensures that the optimal policy is to hold some inventories.

The newsvendor maximizes expected profits from ordering an amount $q$ of the products for upcoming demand. In particular, the newsvendor's optimization problem takes the form:

Newsvendor's Optimization Problem

$$
\max _{q} \mathbb{E}[\pi(q)]=p \int_{0}^{q} x \partial F(x)+p q \int_{q}^{\infty} \partial F(x)-w q,
$$

where $\pi(q)$ is the profits obtained from holding $q$ units of the product, and the expectation is taken over the possible realizations of demand. ${ }^{5}$

\subsection{The optimal inventory}

The solution to the newsvendor's problem takes the familiar form:

$$
q_{F}=F^{-1}\left(1-\frac{w}{p}\right)
$$

where $q_{F}$ denotes the optimal inventory decision for a newsvendor facing demand conditions, $F$, and $F^{-1}(\cdot)$ is the inverse of the cumulative distribution

\footnotetext{
${ }^{4}$ Investigations of the newsvendor problem with an unknown demand distribution also exist and began with the seminal work of Scarf (1957). For a more developed discussion, see O'Neil et al. (2016) and the references within.

${ }^{5}$ This objective function takes the exact form of the social planner's problem presented in Prescott (1975) in his model of the efficient vacancy rate of hotel rooms. Alternative formulations of the newsvendor model characterize the problem as an expected cost minimization problem with separate "overage" and "underage" costs (e.g., Porteus (2002)).
} 
function (e.g., $\left.F^{-1}(y)=\inf \{x: F(x) \geq y\}, y \in[0,1]\right)$. The optimal inventory is characterized by the well-known critical fractile (Arrow et al., 1951), $1-\frac{w}{p}$. It is easy to see that demand conditions that first order stochastically dominate another will yield higher inventory levels, holding the retail and wholesale prices constant.

\subsection{Ordering the uncertainty of demand: The disper- sive order}

To characterize the main results, I use the dispersive order. The dispersive ordering of distributions is a partial ordering that relates to the distance between any pair of quantiles (Saunders, 1978; Saunders and Moran, 1978; Shaked, 1982). Specifically, the dispersive ordering is defined as the following:

Definition 1. A pair of distributions $F$ and $G$ are said to be ordered accord-

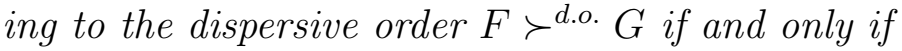

$$
F^{-1}(\beta)-F^{-1}(\alpha) \geq G^{-1}(\beta)-G^{-1}(\alpha)
$$

for any $0<\alpha<\beta<1$.

The dispersive order condition is stronger than both second order stochastic dominance and ordering distributions by their variance. It is a "location invariant" ranking of dispersion, unlike measures of the second moment which depend on the location of the first moment. Many families of distributions exhibit natural orderings in the dispersive order. For example, Saunders and Moran (1978) show that within the family of Gamma distributions, distributions are ordered in the dispersive order according to their "shape" parameter.

\section{Demand uncertainty comparative statics}

The main results entail showing comparative statics for three endogenous values: (i) optimal inventory, $q$; (ii) expected units sold, $\bar{x}$; and (iii) the expected share of inventories sold, $\bar{x} / q$. The primary objective of this paper is to show the implications of changes in the uncertainty of demand, holding other factors constant. To these ends, I make a restriction on the types of distributions that are compared. 
Assumption 2. Any pair of distributions $F$ and $G$ considered are continuous, symmetric, have the same mean (median), and have their domain be on $[0, \infty]$.

Assumption 2 facilitates an easier interpretation of the comparative static results. More specifically, it allows for the analysis to be void of the confounding effects shifts of the center or skewness of the distribution would have had on the incentives for the newsvendor to hold inventory.

The first comparative static involves the optimal inventory decision of the newsvendor.

Proposition 1. Assume the newsvendor faces the optimization problem given by equation 1. Assume distribution functions $F$ and $G$ satisfy assumption 2,

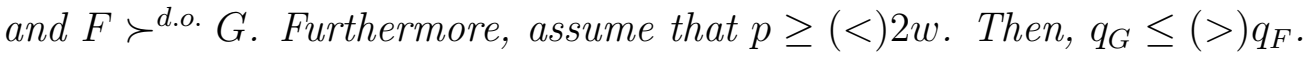

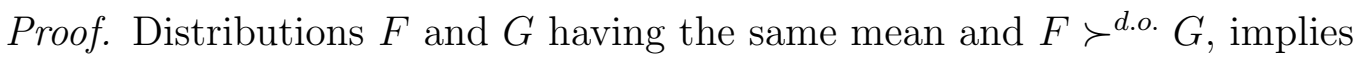
$F$ and $G$ satisfy the single crossing property and that this point must be at the common mean (median) of both distributions (Shaked, 1982, Theorem 2.1). Furthermore, Theorem 2.1 of Shaked (1982) shows that $G$ will cross $F$ from below at the point of intersection. Together these implications yield:

$$
\begin{aligned}
q_{G} & =G^{-1}\left(1-\frac{w}{p}\right) \\
& \leq F^{-1}\left(1-\frac{w}{p}\right)=q_{F}
\end{aligned}
$$

where the inequality follows from the single crossing property and that $p \geq$ $2 w$, which ensures the critical fractile $\geq 1 / 2$. The reverse inequality can be shown similarly in the case that the condition involving $p$ and $w$ is reversed.

The ordering given in Proposition 1 is a well-established result in the newsvendor model literature (e.g., Gerchak and Mossman (1992)). I state it here to illustrate that the dispersive order condition used for the next two results does not lead to a monotone comparative static for the optimal inventory decision.

Intuitively, this result is driven by the non-linearity of the profit function that is imparted by the inventory decision. This creates a kink in the profit function over realizations of demand (Kanbur, 1982). The effect of this kink is similar to valuing a put option, and prevents differentiation techniques 
from being viable (e.g., Rothschild and Stiglitz (1971), Diamond and Stiglitz (1974)).

The next comparative static involves how the expected number of units sold, $\bar{x}$, is influenced by the level of demand uncertainty facing the newsvendor. To ease the exposition, the critical fractile is denoted by $t \equiv 1-\frac{w}{p}$.

Proposition 2. Assume the newsvendor faces the optimization problem given by equation 1. Assume distribution functions $F$ and $G$ satisfy assumption 2,

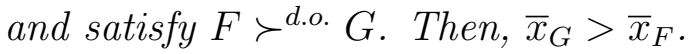

Proof. In the case where $p<2 w$, this result is a consequence of $F$ and $G$ satisfying the single crossing condition where $G$ will cross $F$ from below at the median (of both distributions), and Proposition 1. Because $p<2 w$, the optimal inventory under both the $F$ and $G$ distribution will be below the median (mean) of the distributions, and $q_{G}>q_{F}$. Consequently, the censoring of the demand distribution will occur before the point that $G$ crosses $F$ (from below), and the censoring under $G$ will occur at higher demand levels than the censoring under $F$. Thus, the censored version of $G$ will first order stochastic dominate the censored version of $F$, ensuring $\bar{x}_{G}>\bar{x}_{F}$.

Next, for the case where $p \geq 2 w$, note $h(y) \equiv G^{-1}(y)-F^{-1}(y)$ is decreasing in $y$ by $F \succ^{\text {d.o. }} G$. Furthermore, $F$ and $G$ having the same mean implies that:

$$
\begin{aligned}
0 & =\int_{0}^{1} G^{-1}(y) d y-\int_{0}^{1} F^{-1}(y) d y \\
0 & =\int_{0}^{t} h(y) d y+\int_{t}^{1} h(y) d y<\int_{0}^{t} h(y) d y+\int_{t}^{1} h(t) d y
\end{aligned}
$$

where the inequality follows from $h(y)$ being a decreasing function in $y$. Rearranging the inequality $\left(0<\int_{0}^{t} h(y) d y+\int_{t}^{1} h(t) d y\right)$ yields,

$$
\begin{aligned}
\bar{x}_{G} & =\int_{0}^{t} G^{-1}(y) d y+\int_{t}^{1} G^{-1}(t) d y \\
& >\int_{0}^{t} F^{-1}(y) d y+\int_{t}^{1} F^{-1}(t) d y=\bar{x}_{F} .
\end{aligned}
$$


Unlike in the case of the inventory decision, the expected number of units sold, taking into account the censoring induced by the optimal inventory decision, has a monotone comparative static in the level of the uncertainty of demand.

The dispersive order is a sufficient, but not a necessary condition for the expected number of units to be ordered for all values of $p, w$. A necessary condition for this monotone comparative static requires the final inequality in the proof above holds for all $t$. Though similar to ranking distributions by second order stochastic dominance, the additional influence of the censored demand terms (e.g., $\int_{t}^{1} G^{-1}(t) d y$ ) makes this a stronger condition.

The final comparative static examines how the expected share of inventories sold, $\bar{x} / q$, relates to the level of demand uncertainty.

Proposition 3. Assume that the newsvendor faces the optimization problem given by equation 1 , and that distribution functions $F$ and $G$ satisfy assumption 2, and $F \succ^{\text {d.o. }} G$. Then, $\bar{x}_{G} / q_{G}>\bar{x}_{F} / q_{F}$.

Proof. In Proposition 1, the optimal inventory decisions $q_{G}$ and $q_{F}$ can be ordered based on a condition involving the retail and wholesale prices $(p \geq$ $(<) 2 w)$. In Proposition 2, it was shown that the expected number of units sold must satisfy $\bar{x}_{G}>\bar{x}_{F}$. For the case that $p \geq 2 w, \bar{x}_{G} / q_{G}>\bar{x}_{F} / q_{F}$ follows directly from combining these results.

For the case where $p<2 w$, it can be shown that

$$
\begin{aligned}
\bar{x}_{F} / q_{F} & =\frac{q_{F}-\int_{0}^{t}\left[q_{F}-F^{-1}(y)\right] d y}{q_{F}} \\
& =1-\frac{\int_{0}^{t}\left[q_{F}-F^{-1}(y)\right] d y}{q_{F}} \\
& <1-\frac{\int_{0}^{t}\left[q_{G}-G^{-1}(y)\right] d y}{q_{G}}=\bar{x}_{G} / q_{G}
\end{aligned}
$$

where the inequality follows from the fact that $\left[q_{F}-F^{-1}(y)\right] \geq\left[q_{G}-G^{-1}(y)\right]$ for all $y \in(0, t)$. To show this inequality it is enough to note that $q_{G}-q_{F} \leq$ $G^{-1}(y)-F^{-1}(y)=h(y)$ at $y=t$ and that $h(y)$ is decreasing in $y$ (by $\left.F \succ^{\text {d.o }} G\right)$.

Just like Proposition 2, but unlike Proposition 1, the monotone comparative static involving the expected share of inventories sold is invariant to 
the retail and wholesale prices. Thus, even though the optimal response on inventories to a change in the level of demand uncertainty can go in either direction, the expected share of inventories sold always falls as the amount of demand uncertainty increases.

The model abstracts from many factors likely to impact demand fluctuations including competition, vertical contracts, and inventory costs. Some of these factors-if observed, can be incorporated into the components that comprise the critical fractile (e.g., the retail/wholesale price here). This added flexibility allows for structural estimation approaches akin to Olivares et al. (2008) to elicit differences in the level of uncertainty of demand while controlling for these additional factors.

\section{Conclusion}

I show that for distributions of demand ordered in the dispersive order, both the expected sales and share of inventory sold are ordered. Both of these results hold despite the inventory choice not being ordered. Future empirical work should leverage the robust predictions of observed utilization and (censored) demand to elicit differences in the underlying demand uncertainty. Additionally, exploring how ranking the uncertainty of demand through the dispersive order might extend to dynamic inventory environments is left for future work.

\section{Acknowledgements}

I would like to thank without implicating, an anonymous referee and Joseph E. Harrington, the editor, for many useful suggestions, as well as seminar participants at Indiana University, and Rick Harbaugh for helpful comments, and the generous funding of the Kelley School of Business at Indiana University. 


\section{References}

Andersson, J., Jörnsten, K., Nonås, S. L., Sandal, L., Ubøe, J., 2013. A maximum entropy approach to the newsvendor problem with partial information. European Journal of Operational Research 228 (1), 190-200. URL http://www.sciencedirect.com/science/article/pii/S0377221713000787

Arrow, K. J., Harris, T., Marschak, J., 1951. Optimal inventory policy. Econometrica 19 (3), pp. 250-272.

URL http://www.jstor.org/stable/1906813

Athey, S., 2002. Monotone comparative statics under uncertainty. The Quarterly Journal of Economics 117 (1), 187-223.

URL http://www. jstor.org/stable/2696486

Dana, Jr, J., 1999. Equilibrium price dispersion under demand uncertainty: The roles of costly capacity and market structure. The RAND Journal of Economics 30 (4), pp. 632-660.

URL http://www.jstor.org/stable/2556068

DellaVigna, S., Gentzkow, M., 06 2019. Uniform pricing in US retail chains. The Quarterly Journal of Economics.

URL https://doi.org/10.1093/qje/qjz019

Diamond, P. A., Stiglitz, J. E., 1974. Increases in risk and in risk aversion. Journal of Economic Theory 8 (3), 337 - 360.

URL http://www.sciencedirect.com/science/article/pii/0022053174900908

Eden, B., 1990. Marginal cost pricing when spot markets are complete. Journal of Political Economy 98 (6), pp. 1293-1306.

URL http://www.jstor.org/stable/2937759

Edgeworth, F. Y., 1888. The mathematical theory of banking. Journal of the Royal Statistical Society 51 (1), 113-127.

URL http://www. jstor.org/stable/2979084

Eeckhoudt, L., Gollier, C., Schlesinger, H., 1995. The risk-averse (and prudent) newsboy. Management Science 41 (5), 786-794.

URL http://www. jstor.org/stable/2633098 
Fisher, M., Raman, A., 1996. Reducing the cost of demand uncertainty through accurate response to early sales. Operations Research 44 (1), 8799.

URL http://www.jstor.org/stable/171907

Gaynor, M., Anderson, G. F., 1995. Uncertain demand, the structure of hospital costs, and the cost of empty hospital beds. Journal of Health Economics 14 (3), 291 - 317.

URL http://www.sciencedirect.com/science/article/pii/0167629695000042

Gerchak, Y., Mossman, D., 1992. On the effect of demand randomness on inventories and costs. Operations Research 40 (4), 804-807.

URL http://www . jstor.org/stable/171010

Herweg, F., 2013. The expectation-based loss-averse newsvendor. Economics Letters 120 (3), 429-432.

URL http://www. sciencedirect.com/science/article/pii/S0165176513002723

Kanbur, S. R., 1982. Increases in risk with kinked payoff functions. Journal of Economic Theory 27 (1), 219-228.

URL http://www.sciencedirect.com/science/article/pii/0022053182900242

Kirshner, S. N., Shao, L., 2019. The overconfident and optimistic pricesetting newsvendor. European Journal of Operational Research 277 (1), $166-173$.

URL http://www. sciencedirect.com/science/article/pii/S0377221719301511

Natarajan, K., Sim, M., Uichanco, J., 2017. Asymmetry and ambiguity in newsvendor models. Management Science 64 (7), 3146-3167.

Ninh, A., Hu, H., Allen, D., Apr 2019. Robust newsvendor problems: Effect of discrete demands. Annals of Operations Research 275 (2), 607-621.

URL https://doi.org/10.1007/s10479-018-3016-7

Olivares, M., Terwiesch, C., Cassorla, L., 2008. Structural estimation of the newsvendor model: An application to reserving operating room time. Management Science 54 (1), 41-55.

URL http://www.jstor.org/stable/20122359

O’Neil, S., Zhao, X., Sun, D., Wei, J. C., 2016. Newsvendor problems with demand shocks and unknown demand distributions. Decision Sciences 
$47(1), 125-156$.

URL https://onlinelibrary.wiley.com/doi/abs/10.1111/deci.12187

Petruzzi, N. C., Dada, M., 1999. Pricing and the newsvendor problem: A review with extensions. Operations Research 47 (2), 183-194.

URL http://www.jstor.org/stable/223038

Porteus, E. L., 2002. Foundations of Stochastic Inventory Theory. Standord Business Books.

Prescott, E. C., 1975. Efficiency of the natural rate. Journal of Political Economy 83 (6), pp. 1229-1236.

URL http://www.jstor.org/stable/1830858

Rothschild, M., Stiglitz, J. E., 1971. Increasing risk II: Its economic consequences. Journal of Economic Theory 3 (1), 66-84.

URL http://www.sciencedirect.com/science/article/pii/0022053171900342

Salinger, M., Ampudia, M., 2011. Simple economics of the price-setting newsvendor problem. Management Science 57 (11), 1996-1998.

URL http://www.jstor.org/stable/41262057

Saunders, I. W., 1978. Locating bright spots in a point process. Advances in Applied Probability 10 (3), 587-612.

URL http://www.jstor.org/stable/1426636

Saunders, I. W., Moran, P. A. P., 1978. On the quantiles of the Gamma and F distributions. Journal of Applied Probability 15 (2), 426-432.

URL http://www. jstor.org/stable/3213414

Scarf, H. E., 1957. A min-max solution of an inventory problem. RAND Corporation, 1-15.

URL https://www.rand.org/pubs/papers/P910.html

Shaked, M., 1982. Dispersive ordering of distributions. Journal of Applied Probability 19 (2), pp. 310-320.

URL http://www.jstor.org/stable/3213483

Whitin, T. M., 1955. Inventory control and price theory. Management Science 2 (1), 61-68.

URL http://www.jstor.org/stable/2627238 